C. E. Skinner et T. J. Murray. Journal Infect. Dis., 1924, 34, 585.

S. B. Thomas, E. P. Davies, R. Brown, et K. Elson. Unpublished date (Renseignements non publiés), 1953.

S. B. Thomas, J. M. Harcombe et K. Elson. Unpublished data (Renseignements non publiés, 1954.

W. D. Tiedeman et S. E. Smith, Journal Milk Tech., 1945, 8, 323.

F. O. Tonnex et R. E. Noble. Journal Amer. Wat. Wks Ass., 1930, 22, 488.

F. O. Tonney et R. E. Noble. Journal Bact., 1932, 23, 473.

G. S. Wilson, R. S. Twigg, R. C. Wright, C. B. Hendry, M. P. Cowell, et I. MAIER. "The Bacteriological Grading of Milk" (Le classement bactériologique du lait). Spec. Rep. Ser. Med. Res. Coun., 1935, 206. London : H. M. Stationery Office.

M. W. YALE. Amer. Journal publ. Hlth., 1937, 27, 564.

\title{
LE REFROIDISSEMENT DU LAIT A LA PRODUCTION (Suite et fin)
}

\author{
par \\ Michel ANQUEZ \\ Ingénieur principal du Génie Rural \\ Chef-adjoint à la Section technique du froid.
}

Appareils utilisant la glace.

L'eau du bac peut aussi être refroidie avec de la glace, l'appareil comprend un compartiment spécial où sont placés les pains de glace. Au moment de la traite, ce compartiment est mis en communication avec le bac à eau glacée dans lequel sont plongés les bidons. La température du lait atteint alors, dans des appareils convenablement dimensionnés, $10^{\circ}$ après la première heure, $4^{\circ}$ après la $2^{\mathrm{e}}$ heure. Ces appareils, qui ont été mis au point dans la région de Bordeaux, sont munis d'un compartiment à denrées (fig. 9).

Le compartiment à glace est calculé en fonction de la quantité de lait à refroidir : pour un appareil recevant 30 à 40 litres de lait, il faut prévoir 25 kilogrammes de glace; pour un meuble destiné à refroidir 120 litres de lait, 50 kilogrammes de glace suffisent. La consommation de glace est, en effet, fonction de la capacité de l'appareil ; elle varie de 800 grammes par litre de lait pour un petit meuble à 400 grammes pour un appareil de grand modèle. Ces chiffres tiennent compte du refroidissement des denrées.

Ce procédé implique un ravitaillement facile et régulier en glace, qui peut provenir, soit d'une fabrique de glace existant dans une localité proche, soit d'une installation créée en annexe de l'usine laitière. La distribution de la glace peut d'ailleurs présenter plusieurs aspects. 
Le ravitaillement peut être quotidien, le transport de glace étant assuré par le ramasseur de lait. A la ferme, les pains peuvent être conservés dans une enceinte isotherme annexe et n'être mis dans l'eau du bac où sont immergés les bidons qu'au moment de la

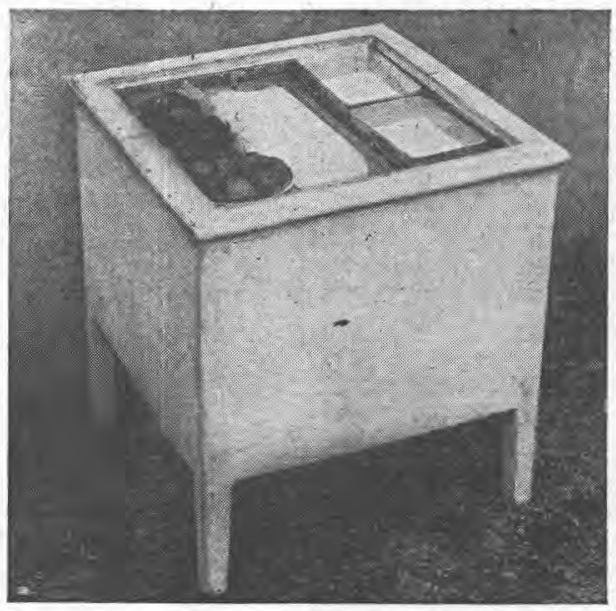

Fig. 9. - Bac mixte pour refroidissement du lait à la ferme et conservation des denrées.

traite. On peut aussi, comme c'est le cas de l'appareil décrit plus haut, mettre la glace dans un compartiment renfermant l'eau de fusion des pains précédents; ce compartiment est séparé du bac à bidons par une simple tôle qu'il suffit d'enlever pour assurer l'intercommunication entre les deux enceintes. Enfin, la glace peut être plongée, dès réception, dans l'eau où seront immergés les bidons.

Le ravitaillement peut aussi être hebdomadaire, ce qui implique l'installation d'une enceinte fortement isolée pour pouvoir conserver la glace.

\section{Aspersion}

\section{Principe.}

Cette technique, mise au point plus tard que les deux autres, consiste à refroidir le lait en faisant ruisseler de l'eau, fraîche ou refroidie, sur les parois des bidons. Il existe des appareils permettant de ne refroidir qu'un seul pot à la fois et d'autres qui sont étudiés pour des litrages plus importants. 


\section{Appareils individuels à turbine (fig. 10, 11).}

Ces appareils se posent sur les pots de lait. L'eau fraîche, qui est ici l'agent réfrigérant, est admise à faible pression dans une turbine qui entraîne un agitateur rotatif plongeant dans le lait;

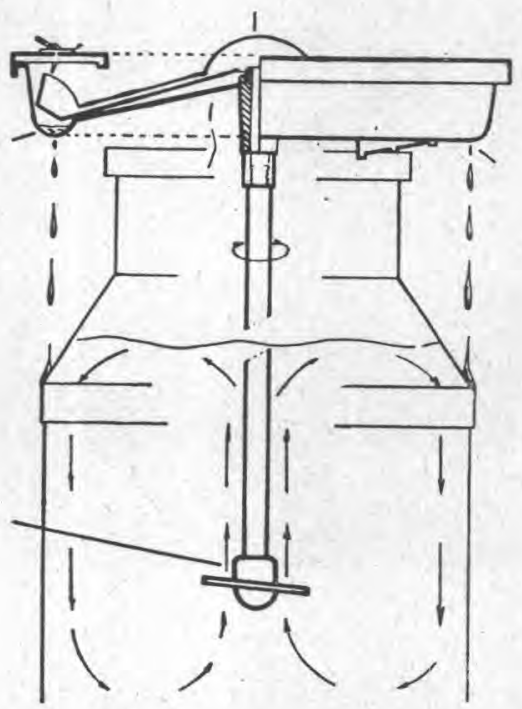

Fig. 10. - Schéma d'un appareil individuel à aspersion avec turbine.

l'eau ruisselle ensuite en nappe mince sur la paroi extérieure du bidon. L'agitateur peut être constitué par une simple hélice ou par un tube lisse parcouru également par l'eau fraîche.

Ces petits appareils sont efficaces (fig. 8 partie gauche) : ils permettent d'abaisser en 15 à 20 minutes, la température du lait à une température supérieure de 2 à $3^{\circ}$ à celle de l'eáu. La consommation d'eau est de 5 à 6 litres par litre de lait. Un seul appareil suffit pour une petite exploitation, car il permet, grâce à la rapidité du refroidissement, de suivre le rythme de la traite. Néanmoins, ce système qui a évidemment l'avantage d'être extrêmement économique présente certains inconvénients : comme tous les appareil à eau fraîche, il ne permet généralement pas de refroidir le lait à la température de $12^{\circ}$, reconnue comme optium par les techniciens ; il faut conserver les bidons rafraîchis, dans une enceinte froide ; enfin, l'appareil doit être nettoyé très soigneusement après chaque opération. En bref, on retrouve les inconvénients des appareils à ruissellement à eau fraîche. 


\section{Appareils collectifs.}

Pour des litrages plus importants, d'autres types, utilisant aussi le principe de l'aspersion ont été mis sur le marché; mais tous emploient de l'eau artificiellement refroidie. Les uns se présentent

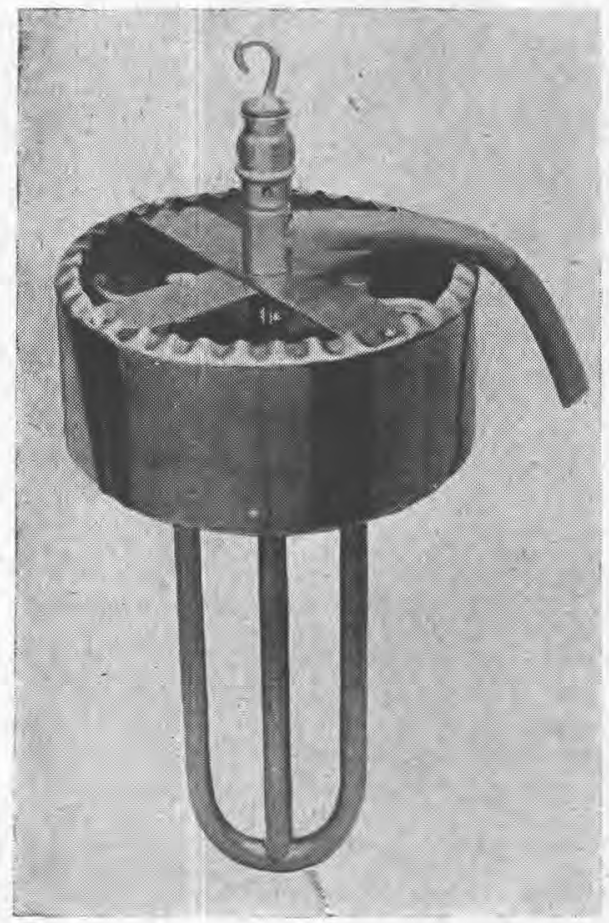

Fig. 11. - Réfrigérant individuel à turbine (ici l'agitateur ereux est parcouru par de l'eau froide).

sous forme d'un meuble complètement isolé (fig. 12), muni de deux portes sur la façade, sans montant au milieu, de façon à introduire facilement les bidons ; dans ce cas, à la partie inférieure de l'appareil se trouve un bac à eau glacée dans lequel plonge la partie inférieure des pots : pour une armoire traitant 400 litres de lait, cette "réserve " d'eau est de 500 litres. Grâce à l'isolation et à la réserve d'eau, le lait peut être maintenu au froid après que la température ait été abaissée au niveau recherché. D'autres appareils sont encore plus simples (fig. 13); dans le but de réduire les frais de premier établissement, le coffre isolé a été supprimé et remplacé par un rideau de nylon; il va de soi que le problème de la conservation est moins aisé à résoudre; dans ce dernier cas aussi, la réserve d'eau 
froide se trouve dans un réservoir spécial. Mais tous ces types ont un même système d'aspersion. Des colliers en caoutchouc ou en matière plastique, percés de trous, sont placés sur l'épaulement

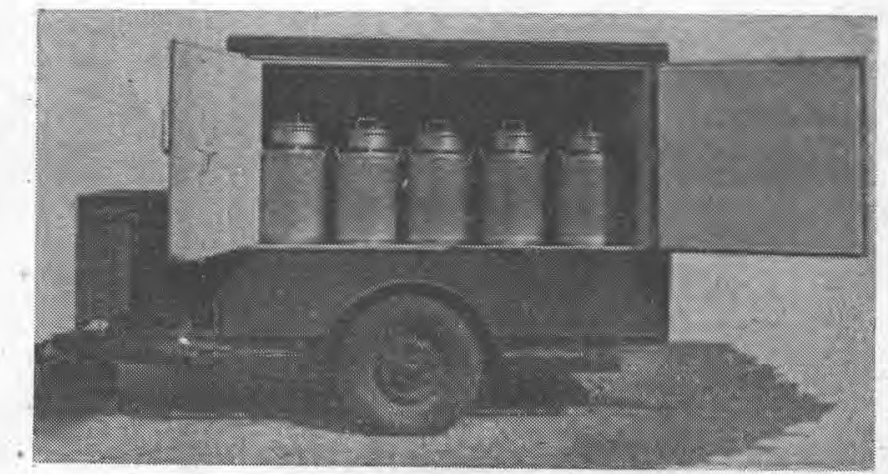

Fig. 12. - Appareil collectif à aspersion. Cet appareil, mobile, comporte une armoire isolée.

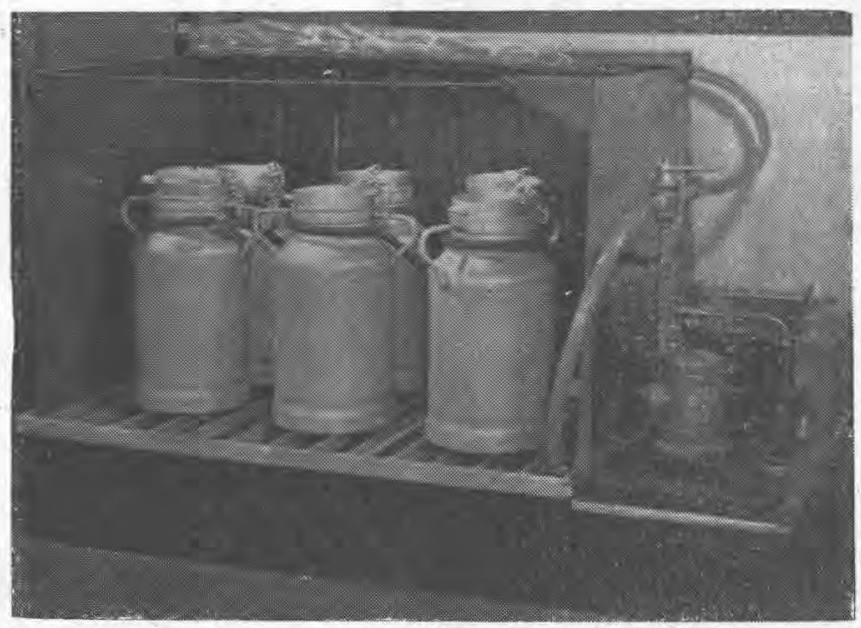

Fig. 13. - Appareil collectif à aspersion. Les pots sont simplement protégés par une toile en nylon.

des bidons; l'eau froide est pompée dans la réserve, refoulée dans les colliers, puis ruisselle sur la paroi extérieure des pots.

Les résultats obtenus sont très satisfaisants, surtout avec les appareils possédant un coffre isolé. Dans le bassin de Dijon, où certains centres de groupage sont ainsi équipés, on a pu constater que le refroidissement est rapide, puisque, sans agitation du lait, 
la température passe de $29^{\circ}$ à $10^{\circ} 5$ en 1 heure. Lorsque la température du lait atteint $10^{\circ}$, la pompe de circulation d'eau est arrêtée, et le refroidissement se poursuit lentement, puisque les bidons restent placés dans une ambiance froide. Avec tous ces appareils, un avantage d'un autre ordre est à signaler : les manipulations sont moins pénibles qu'avec les bacs à immersion, dans lesquels il faut placer les bidons ; cette facilité est surtout appréciée lorsqu'il s'agit de bidons de 40 litres. Notons encore que ces appareils peuvent être installés dans n'importe quel local, pourvu qu'on ait à sa disposition le courant force.

\section{Autres Appareills}

La revue des équipements utilisés, en matière de refroidissement du lait à la production, ne serait pas complète si on ne mentionnait pas quelques appareils, peu utilisés jusqu'ici, en France, du moins, mais dont l'intérêt n'est pas négligeable.

\section{Réfrigérant plongeur.}

Un constructeur a cherché à réaliser un évaporateur mobile, pouvant être introduit dans les pots de lait. Cet évaporateur, en acier inoxydable, se présente sous la forme d'un cylindre allongé ; il est relié au groupe par un tuyau flexible, afin de permettre une introduetion facile dans le bidon (fig. 14). Le nettoyage est très facile et un dispositif a été étudié pour aseptiser le réfrigérant lorsqu'il est dans sa position d'attente. En principe, cet évaporateur est monté en parallèle avec un autre serpentin, fixe cette fois, qui refroidit une armoire ménagère. Le lait peut ainsi être refroidi très rapidement (en moins d'une heure) et doit être entreposé, après refroidissement, dans une ambiance convenable; la dépense supplémentaire d'équipement du réfrigérant à lait est relativement réduite.

Mais jusqu'ici, cet appareil est resté, à notre connaissance, à l'état de prototype.

\section{Le "bulk-handling".}

Le mot même indique qu'il s'agit d'une technique anglosaxonne, américaine dans le cas présent. Le terme signifie « manipulation en vrac " : il s'agit en fait, d'une méthode de ramassage spéciale, toute différente de celle que nous connaissons en France; et la réfrigération à la ferme n'est qu'un des éléments du système. Ce système apparu vers 1948 aux Etats-Unis, prend un essor rapide et tend à remplacer les autres techniques. Le lait est envoyé, dès la traite, dans des tanks isolés, dans la double paroi desquels 
circule un fluide frigorigène en détente directe (la plupart du temps du fréon 12). Le lait est refroidi à une température de l'ordre de $3{ }^{\mathrm{e}}$; la vitesse de refroidissement est accélérée grâce à un agitateur

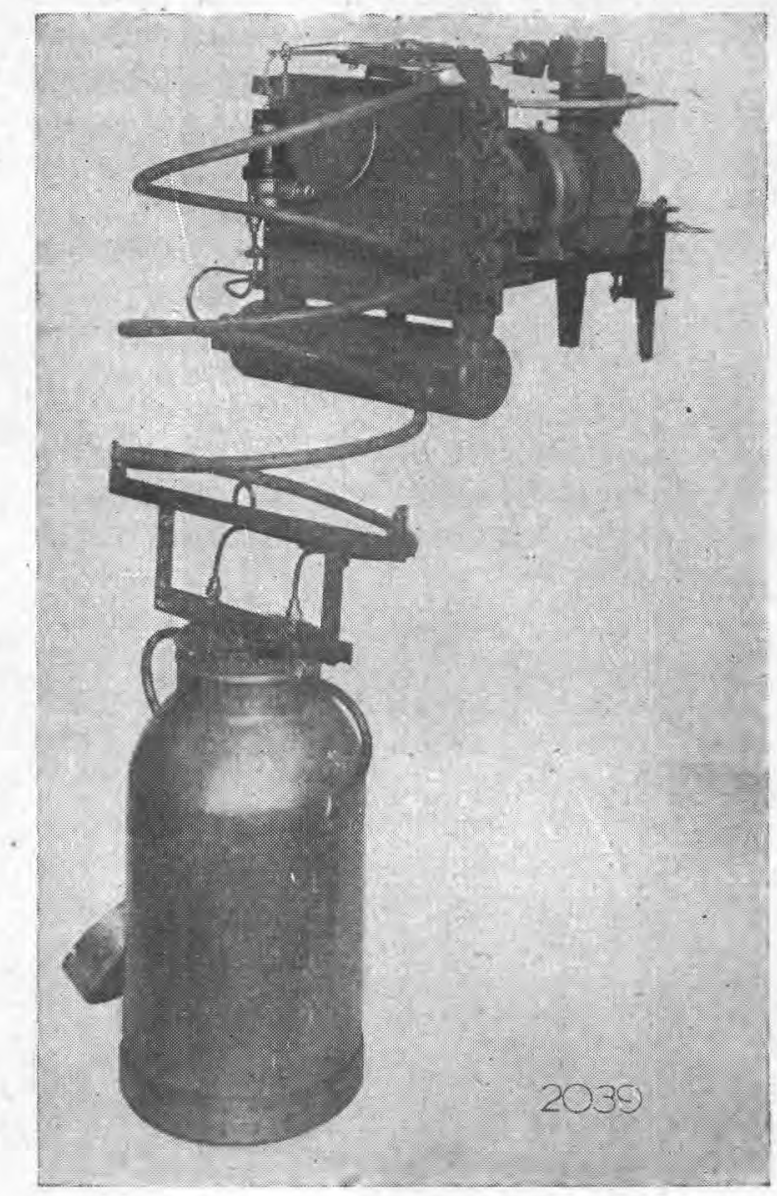

Fig. 14. - Appareil avec évaporateur plongeur.

tournant à"35 tours-minute. Au moment du ramassage, une pompe envoie le lait dans le camion-citerne de la laiterie. Ce système permet de ramasser le lait en plein jour en été, et de ne faire, à certaines saisons, qu'une collecte tous les deux jours. L'expérience américaine montre, en outre, que la qualité du lait est très sensiblement améliorée. 


\section{Problèmes économiques}

La technique du refroidissement du lait à la production semble au point. Un grand nombre de solutions ont été proposées. Et, cependant, il faut bien le dire, aucune des méthodes préconisées n'a reçu jusqu'ici une application généralisée. Certes, on rencontre bien, de-ci, de-là, à l'initiative d'un industriel ou d'une coopérative, des centres qui sont déjà équipés. Mais ces petites "taches" sont encore très disséminées sur l'ensemble du territoire. C'est que le véritable problème n'est pas d'ordre technique, mais économique : alors que des solutions simples ont été trouvées, sur le plan technique il faut bien avouer que les problèmes économiques sont autrement ardus à résoudre.

Après avoir évoqué quelques aspects de lạ complexité de l'étude à entreprendre et avoir donné les caractéristiques générales des solutions types que l'on peut envisager, il restera à essayer de donner des éléments de prix de revient de l'opération.

1. La complexité du problème économique.

La production laitière française, ce n'est un mystère pour personne, est très disséminée. La plupart des exploitations ont moins de 5 vaches laitières : les investissements, même minimes, sont donc très difficiles à réaliser par les producteurs eux-mêmes.

De plus, en France, dans un grand nombre de cas, la population agricole n'est pas groupée ; certes, dans les pays calcaires, ou dans les pays de champs ouverts, la population est concentrée dans les villages; mais, dans toutes les zones de bocages, de champs clos, si propices à la pousse de l'herbe, done à la production laitière, les fermes sont éloignées les unes des autres... Encore un élément qui rend le problème à résoudre plus complexe.

Les essais déjà tentés illustrent d'ailleurs cette complexité! Dans le Soissonnais, où une coopérative équipe ses adhérents, dans le Bordelais, à l'initiative d'un industriel, c'est la conception du refroidissement par immersion qui a prévalu; dans le bassin de Dijon, grâce à une initiative administrative, en Alsace aussi, on a adopté la solution du centre de groupage au village. Dans l'ouest, la Sarthe, plus précisément, on a eu recours à la solution du réfrigérant individuel à turbine. Autant de régions, autant de solutions.

Enfin, il faut constater - et ce n'est pas là un grief - que le producteur n'est pas un philanthrope. Tant qu'une réglementation générale n'imposera pas le paiement du lait à la qualité, il ne sera guère possible d'envisager une extension sensible du refroidissement du lait à la production, puisque le producteur ne retirera aucun 
bénéfice des soins onéreux qu'il donne à son lait. Dans les seules régions où un développement notable a pu être constaté, ce sont les industriels ou les coopératives qui ont créé des primes de qualité, qui permettent alors de rendre rentable, pour le producteur, l'opération de refroidissement.

2. Les solutions-types : refroidissement à la ferme, refroidissement au centre de groupage.

Les expériences réalisées jusqu'ici se classent en deux grandes eatégories : dans certains cas, le refroidissement se fait à la ferme même ; dans d'autres, au contraire, le lait est amené à un centre de groupage où il est refroidi.

Le refroidissement à la ferme s'impose dans les régions d'habitat dispersé. Pour les petites exploitations, l'amortissement des appareils utilisant le froid artificiel paraît prohibitif; la seule méthode possible - et ce serait déjà certes un progrès sérieux semble être celle de l'aspersion, avec utilisation de réfrigérants individuels à turbine. Pour les grandes exploitations au contraire, il semble plus facile d'utiliser des techniques comme le ruissellement ou l'immersion, avec emploi du froid artificiel : une étude d'organisation du travail de la laiterie doit permettre l'équipement de tournées entières de collecte, afin de supprimer, en été, le double ramassage. La technique de l'immersion, plus facile à réaliser au point de vue technique, semble à recommander dans la plupart des cas.

Dans les régions d'habitat groupé, le problème semble plus facile à résoudre, car la solution du "centre de groupage " offre alors un intérêt certain (fig. 15 et 16). L'utilisation de l'eau glacée est possible quelle que soit la méthode de refroidissement employée. Des essais systématiques entrepris dans le bassin laitier de Dijon, il semble résulter que la technique du ruissellement n'est guère intéressante, en raison des lourdes sujétions qu'elle entraîne; par contre, l'immersion et l'aspersion donnent des résultats très intéressants; l'immersion est plutôt à recommander lorsque le litrage collecté ne dépasse pas 400 litres; au-delà, il semble que les appareils à aspersion soient économiquement plus avantageux.

Bien entendu, ces considérations ne doivent pas être prises absolument. En matière économique, tout doit être nuancé. En fait, ce n'est qu'après une étude approfondie de chaque cas particulier que l'on pourra adopter l'une ou l'autre des méthodes.

3. Le prix de revient de l'opération.

Un des éléments de l'étude économique est évidemment le calcul du prix de revient du refroidissement. En la matière, quelques 
expériences ont été faites desquelles il est déjà possible de tirer au moins un ordre de grandeur des dépenses entraînées par ce traitement primaire du lait, aussi bien au point de vue de l'amortissement que de l'exploitation proprement dite.

\section{A. Appareils a Ruissellement}

En utilisant l'eau fraîche, cette technique, qui ne permet pas, dans la plupart des cas, sauf si l'on dispose d'eau exceptionnelle-

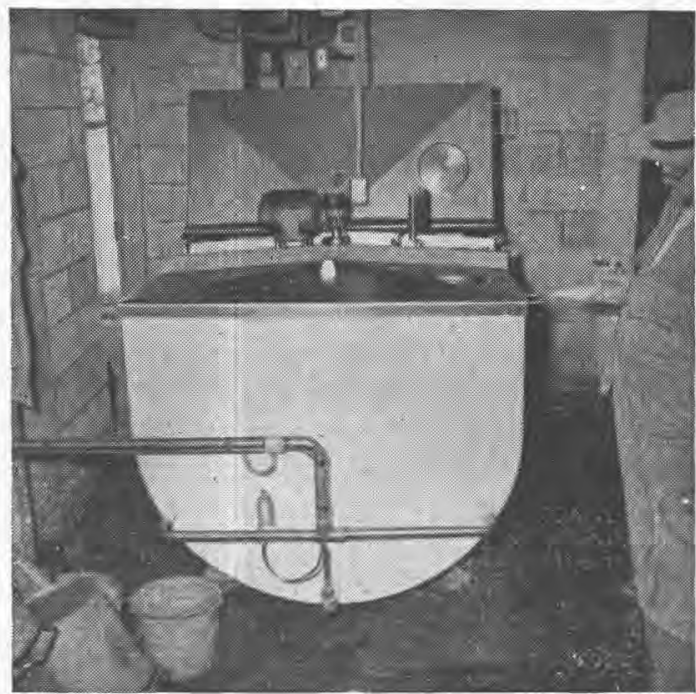

Fig. 15. - "Bulk-handling ».

ment fraîche (7 à $\left.9^{\circ}\right)$, d'obtenir un refroidissement suffisant, est évidemment peu onéreuse. Les réfrigérants à ruissellement ordinaires coûtent environ 50.000 franes ; compte tenu de l'amortissement et du prix de l'eau, le prix de revient varie évidemment suivant l'importance du litrage traité chaque jour : pour un petit producteur traitant 10.000 litres par an, on estime à 1 franc par litre le coût de l'opération; ce coût s'abaisse à 0 fr. 35 environ dans le cas d'une exploitation livrant 75.000 litres par an.

Si l'on utilise l'eau glacée, les résultats techniques sont alors excellents; mais le prix de revient de l'opération s'accroît sensiblement : il est de 2 francs environ dans le cas de la grande exploitation pour atteindre 6 franes dans une petite ferme. Avec des appareils à détente directe, les frais sont sensiblement moins lourds : 
le coût de l'opération ne dépasse pas 0 fr. 85 pour un gros producteur et s'élève à $4 \mathrm{fr}$. 40 pour un petit producteur.

\section{B. Appareils a immersion}

Les appareils à immersion à eau fraîche ne peuvent être recom. mandés qu'en montagne, où la température de l'eau est souvent

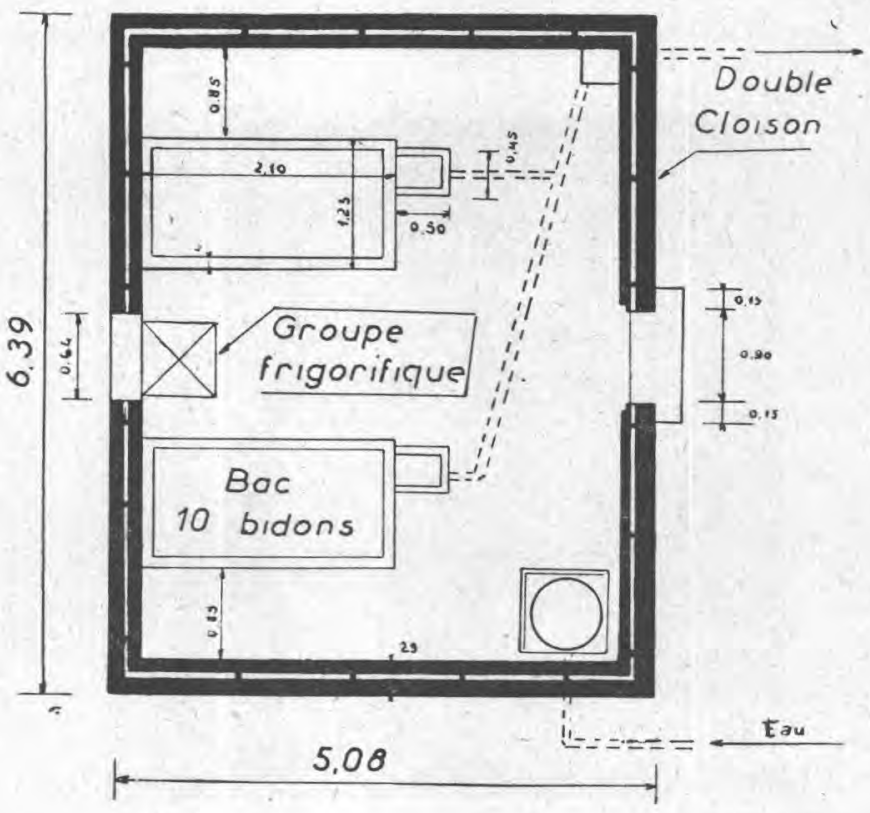

ECHELLE': 0015 m.p.m

Fig. 16. - Plan d'un centre de groupage dans la Côte-d'Or.

très froide. On constate que le prix de revient de l'opération est beaucoup moins fonction du litrage traité que dans le cas précédent. En montagne, où le prix de l'eau est souvent très faible, les dépenses totales, au litre de lait, varient, suivant l'importance de l'exploitation, de 0 fr. 30 à 0 fr. 45.

Les appareils utilisant l'eau glacée, avec groupe frigorifique sont les plus répandus. Les frais d'électricité s'élèvent à $0 \mathrm{fr} .70$ environ par litre de lait; en tenant compte des frais fixes, le prix de revient varie de $1 \mathrm{fr}$. 75 par litre pour une grande ferme laitière à $4 \mathrm{fr} .85$ pour un petit producteur. Dans le bassin laitier de Dijon, le prix de revient, au centre de groupage, s'élève à $3 \mathrm{fr}$. 50 environ par litre ; ce prix, qui peut paraître élevé, s'explique aisément : 
l'amortissement tient compte des dépenses de génie civil qui ont dû être consenties ( 800.000 francs pour le bâtiment, 800.000 francs de matériel) ; d'autre part, le centre ne fonctionne que quatre mois par an pendant lesquels il collecte environ 60.000 litres de lait.

Lorsqu'on emploie la glace, il semble qu'on obtienne des résultats assez intéressants; d'une étude faite dans la région bordelaise, il résulte que les dépenses de glace soient de l'ordre de 1 fr. 50 par litre de lait traité ; l'amortissement des appareils qui traitent environ 100 litres de lait par jour, est évalué également

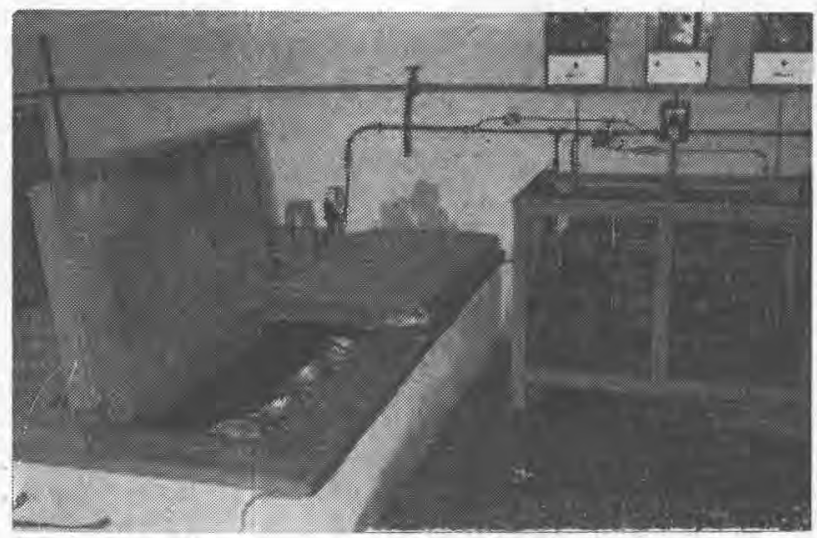

Fig. 17. - Intérieur d'un centre de groupage (Tilchatel).

à 1 franc par litre. Le prix de revient total serait alors de $2 \mathrm{fr}$. 50 environ par litre de lait traité.

\section{Appareils a aspersion}

Les réfrigérants individuels à turbine semblent à préconiser malgré leurs inconvénients, dans les petites fermes des zones d'habitat dispersé, à condition, bien entendu, qu'elles disposent d'eau assez fraîche. Le prix de revient de l'opération ne dépasse pas $0 \mathrm{fr} .50$ par litre.

Les appareils plus importants, utilisant l'eau glacée, sont évidemment d'un prix de revient plus élevé. Pour un centre de groupage de la région dijonnaise, traitant 400 litres par jour et 50.000 litres par an, le prix total de l'opération atteint 3 francs par litre. Ce prix, plus faible que celui entraîné par un appareil à immersion dans un centre de groupage de capacité analogue, tient au fait que les dépenses de génie civil sont beaucoup moins importantes (200.000 francs au lieu de 800.000). Par contre, les frais proportionnels sont sensiblement identiques (0 fr. 85 dans le cas de l'immersion, 0 fr. 95 dans le cas de l'aspersion). 


\section{EXPÉRIENCES ÉTRANGÈRES}

Donnons, en terminant, quelques références étrangères.

On estime, en Angleterre, que le refroidissement du lait à $3^{\circ}$ environ, coûte environ 1/2 penny par gallon, e'est-à-dire environ 0 fr. 50 par litre : il est vraisemblable qu'on ne tient pas compte de l'amortissement.

Aux Etats-Unis, la pratique du bulk-handling reviendrait à 2 francs par litre environ.

\section{CONCLUSION}

Le tour d'horizon que je me proposais de faire est terminé. Cependant, j'ai volontairement passé sous silence un point important des considérations d'ordre économique, il s'agit de la plusvalue qu'on est en droit d'attendre dans l'état actuel des choses, du refroidissement du lait à la ferme. C'est là un problème particulièrement ardu. On peut d'abord souligner que la généralisation d'un tel traitement évite la "tourne", et pendant les mois d'été, l'économie qui en résulte est loin d'être négligeable. Je dois à M. Meriaux, Ingénieur du Génie Rural à Dijon, qui m'a fourni les renseignements relatifs au bassin laitier de cette ville, un chiffre particulièrement significatif. Sur 40.000 litres collectés par jour pour l'agglomération dijonnaise, 5 à 6.000 litres étaient impropres à la consommation ; la création des centres de groupages a supprimé totalement la "tourne»: la plus-value qui en résulte est de l'ordre de 1 fr. 50 par litre. Ajoutons encore que la généralisation du système permettrait d'éviter, dans bien des cas, le double ramassage ; c'est dire qu'il s'en faut de très peu pour que l'opération soit rentable.

\section{TRAVAUX ET RECHERCHES SUR LE LAIT ET LES PRODUITS LAITIERS (1)}

(Suite et $\mathrm{fin}$ )

\section{IV. - Laits conservés (2)}

Depuis le 27 mars 1953, date de la dernière Assemblée du Conseil Scientifique du CNERNA, la Sous-Commission des laits de conserve a tenu quatre réunions :

(1) Centre national de Coordination des Etudes et Recherches sur la nutrition et l'alimentation. Directeur : Professeur Terroine.

(2) Rapport de M. Tapernoux, 1955. 